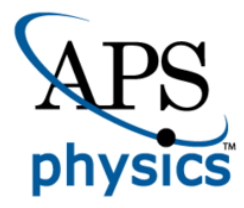

This is the accepted manuscript made available via CHORUS. The article has been published as:

\title{
Observation of Quasichanneling Oscillations
}

T. N. Wistisen, R. E. Mikkelsen, U. I. Uggerhøj, U. Wienands, T. W. Markiewicz, S. Gessner,

M. J. Hogan, R. J. Noble, R. Holtzapple, S. Tucker, V. Guidi, A. Mazzolari, E. Bagli, L.

Bandiera, and A. Sytov (SLAC E-212 Collaboration)

Phys. Rev. Lett. 119, 024801 - Published 13 July 2017

DOI: 10.1103/PhysRevLett.119.024801 


\title{
Observation of Quasi-channeling Oscillations
}

\author{
T.N. Wistisen, R.E. Mikkelsen and U.I. Uggerhøj \\ Department of Physics and Astronomy, Aarhus University, 8000 Aarhus, Denmark \\ U. Wienands, T.W. Markiewicz, S. Gessner, M. Hogan and R.J. Noble \\ SLAC National Accelerator Laboratory, 2575 Sand Hill Road, Menlo Park, California 94025 \\ R. Holtzapple and S. Tucker \\ California Polytechnic State University, San Luis Obispo, California 9340\%, USA \\ V. Guidi, A. Mazzolari, E. Bagli, L. Bandiera and A. Sytov \\ Department of Physics and Earth Sciences of the University of Ferrara, \\ and INFN Section of Ferrara, Via Saragat 1/C, I-44122 Ferrara, Italy
}

\begin{abstract}
We report on the first experimental observations of quasi-channeling oscillations, recently seen in simulations and described theoretically [1. Although above-barrier particles penetrating a single crystal are generally seen as behaving almost as in an amorphous substance, distinct oscillation peaks nevertheless appear for particles in that category. The quasi-channeling oscillations were observed at SLAC National Accelerator Laboratory by aiming $20.35 \mathrm{GeV}$ positrons and electrons at a thin silicon crystal bent to a radius of $R=0.15 \mathrm{~m}$ exploiting the quasi-mosaic effect. For electrons, 2 relatively faint quasi-channeling peaks were observed while for positrons, 7 quasi-channeling peaks were clearly identified.
\end{abstract}

Through the coherent action of the lattice nuclei upon a charged particle penetrating a single crystal along a major crystallographic direction (axis or plane), the particle motion may proceed in a transverse potential barrier and is thus restricted in the transverse direction, the socalled channeling process, see [2] and e.g. [3] for a review. Above-barrier particles, on the other hand, are generally taken as behaving almost identically to interactions in an amorphous substance, a notable exception being coherent bremsstrahlung. It was therefore surprising to learn, first from simulations [1] and as presented here from experiment, that above-barrier particles may actually give rise to oscillation patterns in the angular distribution of the exiting beam. These so-called quasi-channeling oscillations are the main subject of the present paper.

In the absence of inelastic scatterings, the transverse energy is conserved during channeling. Such scattering events may eventually lead to escape (dechanneling) of the particle from the transverse potential well of depth $U_{0}$. Under conditions where the centrifugal force is not excessive, this feature is preserved also in a bent crystal.

The deflection of high energy charged particles in bent crystals is somewhat different for positive and negative particles, with the former detected for the first time in the late 70 's, while the latter only recently [5-7]. The basic phenomenon is shown for a positive particle in figure 1 . depicted in a rotating coordinate system such that the penetration depth $z$ is shown as a straight line. In this figure, all positrons enter the crystal with perfect alignment to the plane, but generally those particles which have an angle of incidence less than the critical angle [2], $\theta_{c}=\sqrt{2 U_{0} / p v}$ may channel, where $p$ and $v$ are the particle momentum and velocity respectively. As can be seen on the crystal potential on the right pane of figure 1, the potential may be somewhat more complicated depending on the crystallographic direction, in this case (111) planes. In the harmonic approximation, mainly but not exclusively [7 - applicable for positively charged particles, the wavelength of the transverse oscillations can be expressed by 8$] \lambda=\pi d \sqrt{p v / 2 U_{0}}$, where $d$ is the inter-planar distance and $U_{0}$ the potential depth.

The bound motion is disturbed by multiple scattering interactions with the crystal atoms that may increase the transverse energy of the particle sufficiently, such that it leaves the channel. Such dechanneling processes occur most likely near the lattice sites in the crystal, after which the particle typically interacts with the crystal as if it were an amorphous medium. In the present case, however, the dechanneled particles leave the crystal while still being influenced by the transverse potential.

Channeled particles at the extreme points in their oscillations have a higher dechanneling probability and the wave-pattern shown in figure 1 may be observed if the depth coordinate at which the dechanneling takes place can be measured. This is exactly what is achieved in a bent crystal, where the crystal angle corresponding to a particular depth of penetration translates into a specific position downstream. Thus, the wave-pattern gives rise to oscillations observed on a downstream screen, the so-called channeling oscillation peaks.

A crystal with thickness $l_{c r}$ and bending angle $\theta_{b}$, may lead to a constant angular distance between any two neighboring channeling peaks of $\Delta \phi_{\mathrm{ch}}=\lambda \theta_{b} / 2 l_{c r}$. Such planar channeling oscillations have been observed at low energy back scattering experiments, see [1] and references therein. 

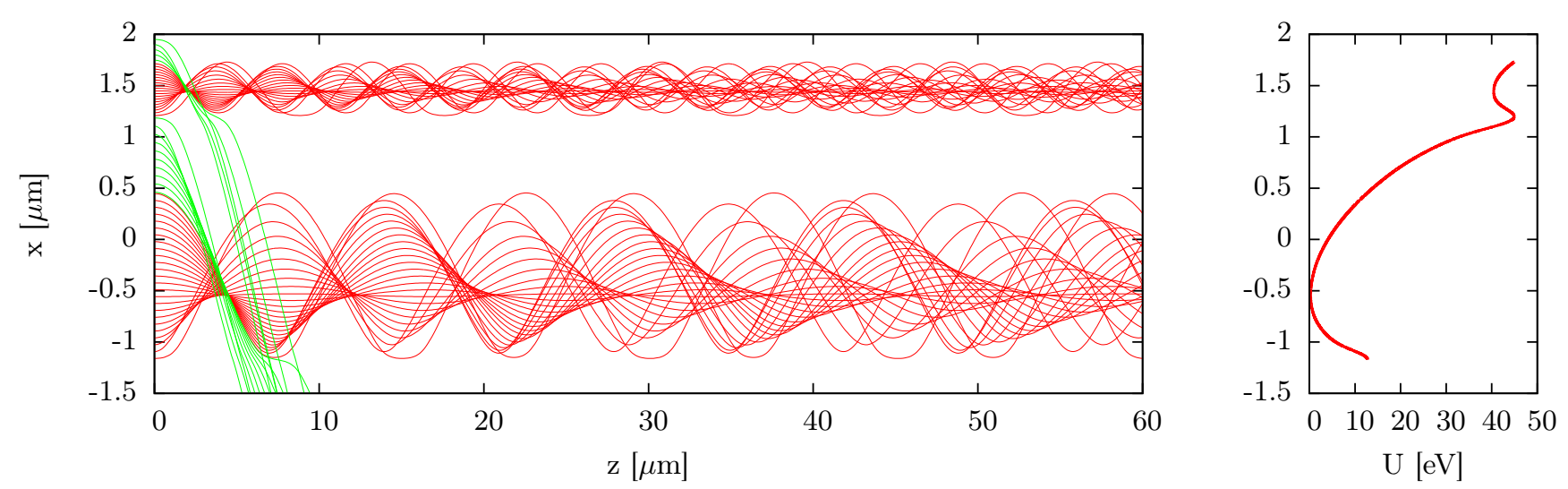

Figure 1. Calculated channeling oscillations. Left: Trajectories of $20.35 \mathrm{GeV}$ positrons penetrating a bent crystal with with radius of curvature $R=0.15 \mathrm{~m}$; where each line corresponds to a different initial transverse coordinate, $x$. Multiple scattering dechanneling is neglected and the incident particle is assumed to be exactly aligned to the planar direction. Red curves are the channeled particles while the green are those not accepted into the channel at the crystal surface. Right: The centrifugally distorted transverse potential energy curve calculated using the Doyle-Turner thermally averaged continuum potential 4 .

On the other hand, similar oscillation patterns, arising from a completely different mechanism, the so-called quasi-channeling oscillation peaks, may also appear [1. These oscillations were treated numerically in a recent study 1] using the CRYSTAL simulation code 9, 10. Like channeling oscillations, this leads to peaks in the deflection spectrum observed from the crystal, but in this case not evenly distributed, and here arising from overthe-barrier motion, see figure 2.

The effect was initially only simulated for $7 \mathrm{TeV}$ protons, but later also for electrons and positrons, and examples were provided at $20.35 \mathrm{GeV}$ beam energy. Particles that are initially channeled, and follow the curvature of the crystal, may dechannel at some point downstream. When this dechanneling occurs, the trajectories become grouped near the region where the transverse kinetic energy is smallest, i.e. the transverse location where the particles spend the most time. This gives rise to distinct peaks in the dechanneling tail, see the green curves in figure 2

The key feature that we will look for in this experiment is the angular location of the quasi-channeling peaks. The center of these can be approximated [1] for electrons by

$$
\theta_{\mathrm{qch}}^{-} \sim \theta_{d}-\sqrt{\frac{2 d_{0} n}{R}}
$$

and for positrons by

$$
\theta_{\mathrm{qch}}^{+} \sim \theta_{d}-\sqrt{\frac{2 d_{0}(n-1)}{R}+\frac{2 d_{s}}{R}}
$$

where $d_{s}=d_{0} / 4$ is the width of the narrow (111) channel, $d_{0}=d_{1}+d_{2}=3.14 \AA$ is the sum of their interplanar distances and $\theta_{d}=\theta_{b}+\theta_{t}$, with $\theta_{t}$ being the crystal angle offset from ideal channeling orientation. From these equations it is seen that the location of the quasichanneling peaks depends only weakly on the particle charge sign and therefore almost solely on crystal characteristics. The two phenomena of channeling peaks vs. quasi-channeling peaks can thus be identified by a different scaling, linear vs. a square root.

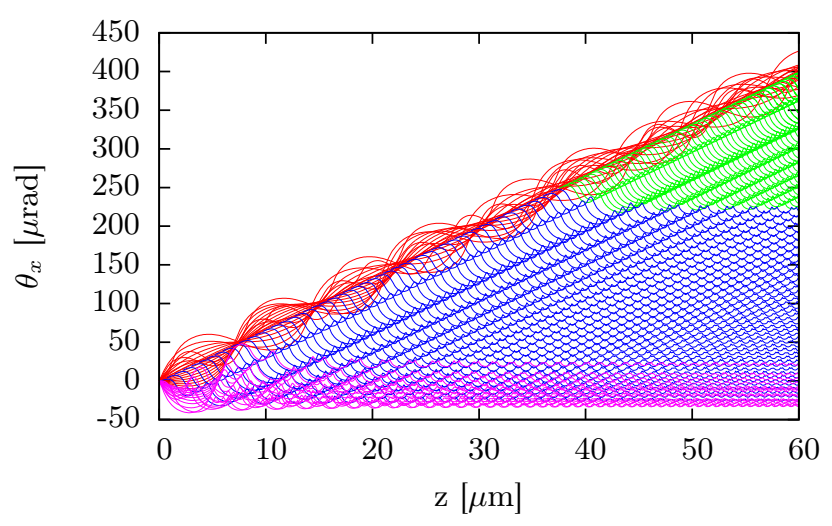

Figure 2. Calculated quasi-channeling oscillations. Angles of positrons as a function of penetration depth in a bent crystal. The initial transverse coordinate, $x$ is fixed to $-1.2 \AA$ for the green and blue curves, mimicking dechanneled particles, see figure 1, right panel. With purple is shown those particles that do not channel initially, due to surface transmission [4, with red those that channel through the entire crystal. Of the dechanneled particles, the green trajectories tend to 'condense' at 7 specific angles $\theta_{x}$ at the exit of the crystal, $z=60 \mu \mathrm{m}$ in accordance with our experimental observation.

The experiments were performed at the SLAC Facility for Advanced Accelerator Experimental Tests (FACET), using beams of $20.35 \mathrm{GeV}$ electrons and positrons respectively. The crystal under study was fabricated at the University of Ferrara in the Sensors and semiconductor 


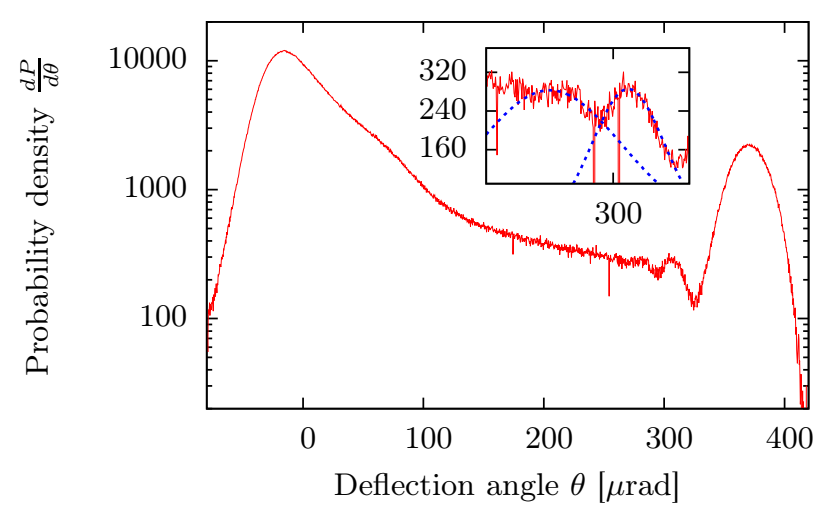

Figure 3. Angular distribution of electrons obtained at maximum channeling conditions. The insert provides a zoom in the regions around the quasi-channeling peaks. The blue curves are individual gauss fits applied to obtain the center of the oscillations. Fits are performed for deflection angles of $0.33-0.36$ mrad and 0.36-0.39 mrad respectively.

Laboratory [11. It is a silicon ( $\mathrm{Si}$ ) crystal with a thickness of $l_{c r}=60 \pm 1 \mu \mathrm{m}$. The crystal bending exploits the quasi-mosaic effect [12] to provide (111) bent planes at a radius $R=15 \pm 0.42 \mathrm{~cm}$. The bending angle of the crystal is $\theta_{b}=402 \pm 9 \mu \mathrm{rad}$. Furthermore, we performed an XRD analysis and measured an anticlastic curvature to a radius not less than $80 \mathrm{~m}$, i.e. almost its complete suppression.

The crystal was mounted on a rotation table that could be moved with roughly $5 \mu$ rad precision such that an angle scan of the channeling phenomenon could be performed. The rotation angle was measured by reflecting a laser beam off the crystal holder and measuring the position of the reflected laser spot as it moved on a screen placed $1.16 \mathrm{~m}$ away.

The spatial resolution of the $e^{ \pm}$detector screen was $3.5 \mu \mathrm{m}$ and its effective area was 7.6 by $8.8 \mathrm{~mm}$.

For electrons, the angular distribution obtained when the crystal is oriented in the maximum channeling direction is shown in figure 3.

The leftmost peak is the primary beam and the smaller rightmost peak are the channeled electrons. At angles slightly below the latter, two quasi-channeling oscillations are visible. As predicted in [1, these oscillations are only visible near the channeling peak. The locations of the two peaks are obtained by fitting gaussian functions separately and the distance between them is $30.5 \pm 2 \mu \mathrm{rad}$. This is somewhat higher than the theoretical estimate for the distance between the quasichanneling peaks, $(\sqrt{2}-1) \sqrt{2 d_{0} / R}=26.8 \mu \mathrm{rad}$, based on equation (1). Results from a simulation taking into account all important experimental parameters yield channeling peak FWHMs of $44 \mu \mathrm{rad}$ for $e^{-}$and $48 \mu \mathrm{rad}$ for $e^{+}$, to be compared with the experimental values of $39 \mu \mathrm{rad}$ and $48 \mu \mathrm{rad}$, respectively. This agreement, though not

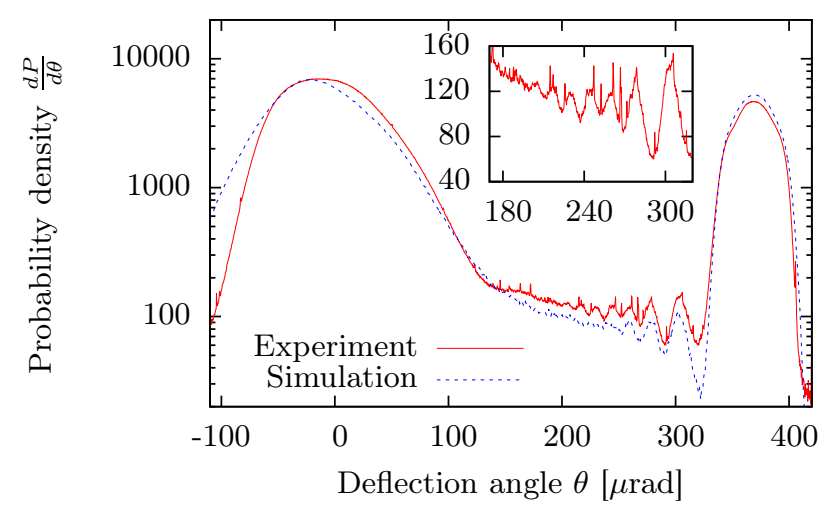

Figure 4. Shown with the red, full-drawn line is the angular distribution as in figure 3. but for positrons, zoomed and taken without the optical attenuator at the detection screen, to optimize for the intensity of dechanneling peaks. The dashed, blue line shows the result of simulations.

perfect due to imperfections in the transverse potential simulation, gives a very convincing support to our measurements.

For positrons at deflection angles of $\sim 0.2-0.36 \mathrm{mrad}$, quasi-channeling oscillations can be clearly seen in the dechanneling tail of the red, full-drawn experimental data line in figure 4 We have zoomed in on the relevant angular region and the data used for this zoom was taken without an optical filter which attenuates the light hitting the camera, such as to optimize for the observation of the channeling oscillations. These quasi-channeling oscillations are primarily obtained when the crystal is oriented in the maximum channeling configuration. Results from simulations, in good overall agreement with experiment, is shown in figure 4 as the blue, dashed line.

The experimental peaks are fitted by individual gaus-

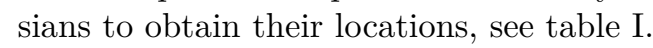

\begin{tabular}{|c||c|c|c|c|c|c|c|}
\hline $\mathrm{n}$ & 2 & 3 & 4 & 5 & 6 & 7 & 8 \\
\hline \hline$\theta_{\text {peak }}(\mu \mathrm{rad})$ & 299 & 275 & 256 & 241 & 226 & 213 & 201 \\
\hline$\sigma_{\text {peak }}(\mu \mathrm{rad})$ & 4.1 & 3.5 & 3.8 & 3.5 & 3.6 & 3.9 & 3.7 \\
\hline
\end{tabular}

Table I. A table showing the positions and uncertainty $(\sigma)$ of the quasi-channeling peaks for positrons.

These locations are then used as an input to a fitting procedure based on equation (2), rewritten as $\theta_{\mathrm{qch}}^{+}=$ $\theta_{d}-a \sqrt{2(n-1)+\frac{1}{2}}$ where $a=\sqrt{d_{0} / R}, n=2, \ldots, 8$ being the peak number. We start from $n=2$ since the $n=1$ peak is hidden inside the peak from the channeled particles.

From the fit we obtain $a_{\exp }=(4.39 \pm 0.06) \times 10^{-5}$ and $\theta_{d}=(368 \pm 1.8)$ urad which is the $95 \%$ confidence bound from the fit. Adding errors from the calibration procedure we obtain $a_{\exp }=(4.39 \pm 0.13) \times 10^{-5}$. The value of 


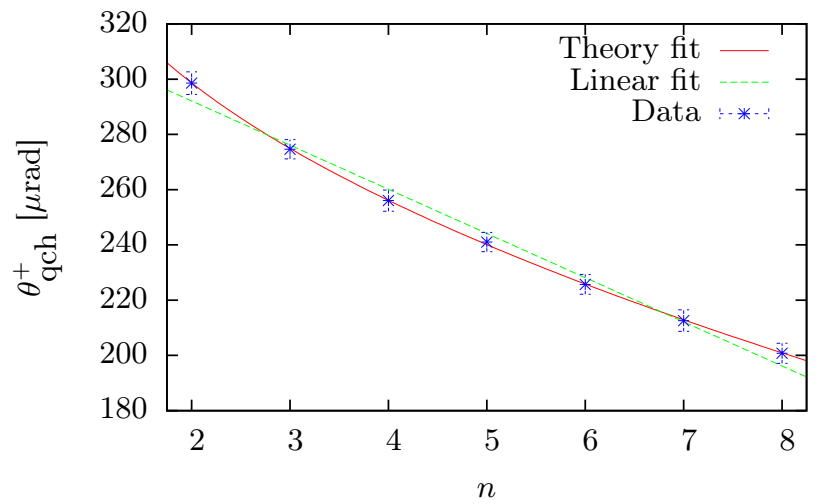

Figure 5. The experimentally obtained positions of the quasichanneling peaks for positrons are shown along with a fit based on equation (2) and a linear fit for comparison.

$\theta_{d}$ fits well with the observed position of the channeling peak in figure 4 of $369 \mathrm{\mu rad}$. Performing a linear fit of the form $\theta_{\mathrm{qch}}^{+}=c n+b$ we obtain $b=(324.1 \pm 12) \mu \mathrm{rad}$ and $c=(-15.99 \pm 2.1)$ urad. The comparison of the fits and data can be seen in figure 5 From equation (2) we would expect $a_{\text {theory }}=(4.57 \pm 0.06) \times 10^{-5}$ where the uncertainty comes from the uncertainty on $R$. Based on this we conclude that theory and measurement show agreement within the experimental uncertainty. In the linear case we would expect $|c|$ to equal $\Delta \phi_{\mathrm{ch}}$, however from theory we have $\Delta \phi_{\mathrm{ch}}=49 \mathrm{prad}$ which is a large discrepancy. As seen in figure 5 the formula of equation (2) captures the scaling better than the linear model as well. This, along with the fact that oscillations in the dechanneling tail for electrons can not be explained by channeling oscillations, since here the motion is far from harmonic as shown for positrons in figure 1 leads us to the conclusion that the observed oscillations must be the quasi-channeling oscillations. In the experiment an absolute measurement of the deflection angle was not possible due to intervening optics in the form of a quadrupole doublet with the optics known to a limited precision. Therefore the deflection angles were calibrated such that the diagonal in a triangle plot, see [6, 7], has a slope of 1 . It should be mentioned that quasi channeling peaks and the volume reflection peak (leftmost in figure 4) are sensitive to beam jitter and this is the source of the errors shown in table $\mathbb{I}$ which were obtained by looking at measurements from 10 bunches.

The presented measurements were performed with a lattice function of $\beta_{x}=\beta_{y}=10 \mathrm{~m}$, leading to a divergence in the deflection plane of approximately $10 \mu \mathrm{rad}$ for $e^{-}$and $20 \mu \mathrm{rad}$ for $e^{+}$, with about a factor $\simeq 2$ systematic uncertainty. For electrons, additional measurements were done for other beam divergences, i.e. betafunctions of $\beta_{x}=\beta_{y}=20 \mathrm{~m}$ and $\beta_{x}=\beta_{y}=100 \mathrm{~m}$. No quasi-channeling oscillations could be observed for these other values of the beta-function for electrons. This is at first sight surprising, since larger beta-functions make the beam more parallel and from theory we would therefore expect the peaks to become more pronounced. However, the size of the beam also increases with increasing betafunctions, which in this setup becomes detrimental to the observation of the quasi-channeling oscillations. The observation reported here thus not only requires a high energy beam of very small emittance, but also a quite accurately tuned beam in terms of lattice parameters.

By measuring the positions of the quasi-channeling peaks, one can extract information for both bent crystal radius, $R$, and orientation $\theta_{t}=\theta_{d}-\theta_{b}=\theta_{d}-l_{c r} / R$ in one single measurement, i.e. for one goniometer position. Indeed, existing methods require the rotation of the goniometer at different positions around channeling to measure the crystal-to-beam orientation and consequent analysis of the beam deflection efficiency, which is not always so straightforward. The knowledge of the position of the quasi-channeling peaks would improve the alignment procedure between the bent crystal planes and the beam direction, even for large misalignment, in high-energy accelerators, where the crystal can be exploited as a passive and inexpensive tool for beam extraction/collimation. Furthermore, with the use of a bent crystal, one may provide a direct measurement of the quasi-channeling oscillation wavelength, in analogy with the channeling one $(\lambda)$, as a function of the distance between quasi-channeling peaks: $\lambda_{q c h}=R \Delta \theta_{q c h}$. Such value may provide an insight to the interplanar potential shape and strength, and on the dynamics of over-barrier particles, thus giving important information for applications in high-energy particle accelerators, for either beam steering or intense electromagnetic radiation generation.

We can conclude that the quasi-channeling oscillations, presented here for the first time in an experiment, are observed at angles in good agreement with expectations.

This work was partially supported by the Danish Council for Independent Research, by the Italian Istituto Nazionale di Fisica Nucleare (INFN) through the CHANEL experiment, in part by the US NSF under contracts PHYS-1068662 and PHYS-1535696 and in part by the U.S. DOE under Contract No. DE- AC02-76SF00515 and by the U.S. National Science Foundation (Grant No. PHY-1068662). We acknowledge the CINECA award under the ISCRA initiative, for the availability of high performance computing resources and support for the simulations.

[1] A. I. Sytov, V. Guidi, V. V. Tikhomirov, E. Bagli, L. Bandiera, G. Germogli, and A. Mazzolari, The European Physical Journal C 76, 77 (2016)

[2] J. Lindhard, K. Dan. Vidensk. Selsk. Mat. Fys. Medd. 34, no. 14, 1 (1965).

[3] U. I. Uggerh $\varnothing$ j, Rev. Mod. Phys. 77, 1131 (2005). 
[4] S. P. Møller, Nuclear Instruments and Methods in Physics Research Section A: Accelerators, Spectrometers, Detectors and Associated Equipment 361, 403 (1995)

[5] W. Scandale, A. Vomiero, S. Baricordi, P. Dalpiaz, M. Fiorini, V. Guidi, A. Mazzolari, R. Milan, G. D. Mea, G. Ambrosi, B. Bertucci, W. Burger, P. Zuccon, G. Cavoto, R. Santacesaria, P. Valente, E. Vallazza, A. Afonin, Y. Chesnokov, V. Maisheev, I. Yazynin, A. Kovalenko, A. Taratin, A. Denisov, Y. Gavrikov, Y. Ivanov, L. Lapina, L. Malyarenko, V. Skorobogatov, V. Suvorov, S. Vavilov, D. Bolognini, S. Hasan, and M. Prest, Physics Letters B 680, 129 (2009).

[6] U. Wienands, T. W. Markiewicz, J. Nelson, R. J. Noble, J. L. Turner, U. I. Uggerhøj, T. N. Wistisen, E. Bagli, L. Bandiera, G. Germogli, V. Guidi, A. Mazzolari, R. Holtzapple, and M. Miller, Phys. Rev. Lett. 114, 074801 (2015)

[7] T. N. Wistisen, U. I. Uggerhøj, U. Wienands, T. W.
Markiewicz, R. J. Noble, B. C. Benson, T. Smith, E. Bagli, L. Bandiera, G. Germogli, V. Guidi, A. Mazzolari, R. Holtzapple, and S. Tucker, Phys. Rev. Accel. Beams 19, 071001 (2016)

[8] V. Biryukov, Y. Chesnokov, and V. Kotov, Crystal Channeling and its Application at High-Energy Accelerators (Springer-Verlag Berlin Heidelberg, 1997).

[9] A. Sytov and V. Tikhomirov, Nuclear Instruments and Methods in Physics Research Section B: Beam Interactions with Materials and Atoms 355, 383 (2015)

[10] E. Bagli, L. Bandiera, V. Guidi, A. Mazzolari, D. De Salvador, A. Berra, D. Lietti, M. Prest, and E. Vallazza, The European Physical Journal C 74, 2740 (2014).

[11] G. Germogli, A. Mazzolari, L. Bandiera, E. Bagli, and V. Guidi, Nuclear Instruments and Methods in Physics Research Section B: Beam Interactions with Materials and Atoms 355, 81 (2015).

[12] R. Camattari, V. Guidi, V. Bellucci, and A. Mazzolari, Journal of Applied Crystallography 48, 977 (2015). 